\title{
Medical Image of the Week: Left Ventricular Non-compaction
}

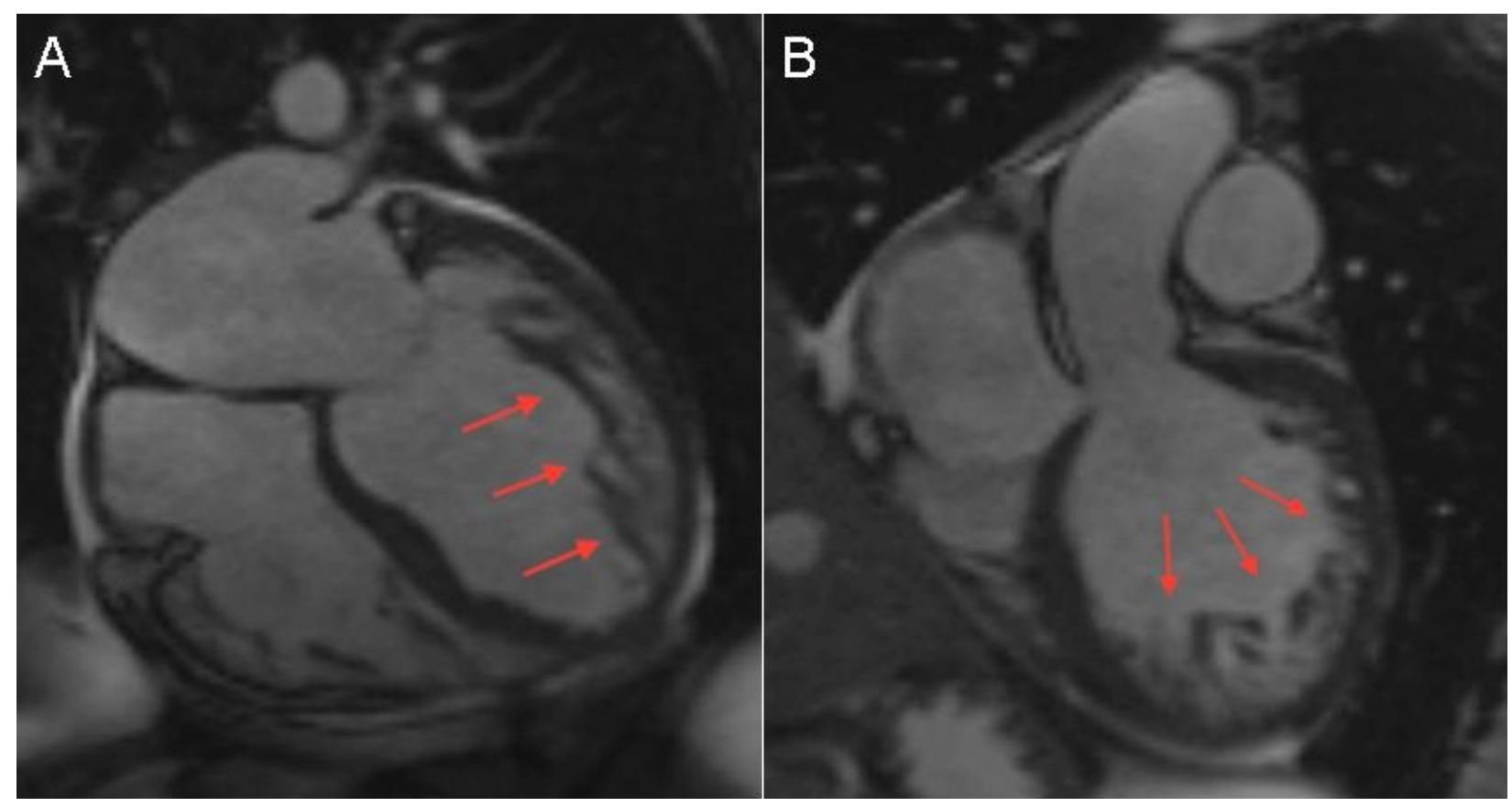

Figure 1. Cardiac MRI showing severely enlarged and remodeled left ventricle (LV) and moderately enlarged right ventricle (RV) with severe global hypokinesis and akinesis of the interventricular septum. Significant trabeculation was noted in the apical, anterolateral and anterior segments of the LV (red arrows), consistent with LV noncompaction.

A 38-year-old woman with history of type 2 diabetes mellitus and hypertension presented to emergency department with worsening exertional dyspnea and orthopnea for the past 2-3 months. She also reported a 14 pound weight gain within the 2 weeks prior to presentation. She denied any prior history of cardiac or pulmonary disease. Also, there was no family history of heart disease. She denies any recent sick contacts, smoking, alcohol drinking, or substance abuse.

Physical exam revealed jugular venous pressure of $10 \mathrm{~cm} \mathrm{H}_{2} \mathrm{O}$ and significant bilateral lower extremity pitting edema. Chest $\mathrm{x}$-ray showed an enlarged cardiac silhouette. Brain naturetic peptide (BNP) was $2,917 \mathrm{pg} / \mathrm{mL}$. A subsequent echocardiogram revealed a left ventricular (LV) ejection fraction of $23 \%$ with severe global LV hypokinesia with moderate mitral regurgitation. Thyroid panel as well as iron panel were within normal range. Other laboratories were unremarkable. For the new onset systolic heart failure, a coronary angiography was performed, which demonstrated normal coronary arteries. The patient was diagnosed with non-ischemic cardiomyopathy and underwent a cardiac MRI, which showed severely enlarged and remodeled LV and moderately enlarged right ventricle (RV) with severe global hypokinesis and akinesis of the intraventricular septum. Moreover, a significant trabeculation was noted in the apical, antero-lateral and anterior segments of the left ventricle (Figure 1), consistent with "LV non-compaction" 
without any evidence of LV thrombus. The patient was started on diuretics and safely discharged with significant symptoms improvement.

LV non-compaction is a cardiomyopathy characterized by altered myocardial wall with prominent left ventricular trabeculae and deep intertrabecular recesses (1). Some authors believe that non-compaction of the ventricular myocardium results from abnormal persistence of the trabecular layer while others believe that altered regulation in cell proliferation, differentiation, and maturation during ventricular wall formation, resulting in hyper-trabeculation (2). Its prevalence in the general population is unknown but among patients undergoing echocardiography is estimated at 0.014 to 1.3 percent. In patients with heart failure, its prevalence has been reported as 3 to 4 percent (3). Patients with LV non-compaction may present with heart failure, arrhythmias, sudden cardiac arrest, syncope, and thromboembolic events. The diagnosis is usually established by transthoracic echocardiography. When echocardiography is indeterminate, cardiac MRI, computed tomography, or left ventriculography could be an alternative diagnostic modality. Data on treatment of LV non-compaction are limited, and there is no standard therapy established for this condition. Medical management depends on the clinical manifestations, LV ejection fraction, presence of arrhythmias, and risk of thromboembolism.

Rostam Khoubyari MD ${ }^{1,2}$ and Seongseok Yun MD PhD ${ }^{3}$

${ }^{1}$ Department of Cardiology, ${ }^{2}$ Sarver Heart Center; and the ${ }^{3}$ Department of Medicine, University of Arizona

Tucson, AZ USA

\section{References}

1. Maron BJ, Towbin JA, Thiene G, et al. Contemporary definitions and classification of the cardiomyopathies: an American Heart Association Scientific Statement from the Council on Clinical Cardiology, Heart Failure and Transplantation Committee; Quality of Care and Outcomes Research and Functional Genomics and Translational Biology Interdisciplinary Working Groups; and Council on Epidemiology and Prevention. Circulation. 2006 Apr 11;113(14):1807-16. [CrossRef] [PubMed]

2. Henderson DJ, Anderson RH. The development and structure of the ventricles in the human heart. Pediatr Cardiol. 2009 Jul;30(5):588-96. [CrossRef] [PubMed]

3. Kovacevic-Preradovic T, Jenni R, Oechslin EN, Noll G, Seifert B, Attenhofer Jost $\mathrm{CH}$. Isolated left ventricular noncompaction as a cause for heart failure and heart transplantation: a single center experience. Cardiology. 2009;112(2):158-64. [CrossRef] [PubMed] 